\title{
The Impact of Quasi-equally Spaced Sensor Layouts on Field Reconstruction
}

\author{
Alessandro Nordio \\ Dip. di Elettronica \\ Politecnico di Torino \\ Torino, Italy \\ alessandro.nordio@polito.it
}

\author{
Carla Chiasserini \\ Dip. di Elettronica \\ Politecnico di Torino \\ Torino, Italy \\ chiasserini@polito.it
}

\author{
Emanuele Viterbo \\ DEIS \\ University of Calabria \\ Cosenza, Italy \\ viterbo@deis.unical.it
}

\begin{abstract}
We consider wireless sensor networks whose nodes are randomly deployed and, thus, provide an irregular sampling of the sensed field. The field is assumed to be bandlimited; a sink node collects the data gathered by the sensors and reconstructs the field by using a technique based on linear filtering. By taking the mean square error (MSE) as performance metric, we evaluate the effect of quasi-equally spaced sensor layouts on the quality of the reconstructed signal. The MSE is derived through asymptotic analysis for different sensor spatial distributions, and for two of them we are able to obtain an approximate closed form expression. The case of uniformly distributed sensors is also considered for the sake of comparison. The validity of our asymptotic analysis is shown by comparison against numerical results and it is proven to hold even for a small number of nodes. Finally, with the help of a simple example, we show the key role that our results play in the deployment of sensor networks.
\end{abstract}

\section{Categories and Subject Descriptors}

C.2.1 [Computer Systems Organization]: Computer-Communication Networks Network Architecture and Design; G.1.0 [Mathematics of computing]: Numerical Analysis General; G.3 [Mathematics of computing]: Probability and Statistics

\section{General Terms}

Performance, Theory

\section{Keywords}

Signal reconstruction, Sensor networks, Irregular sampling, Performance analysis

\section{INTRODUCTION}

The reconstruction of bandlimited, regularly sampled (or equally spaced sampled) signals is a very deeply understood technique; on the contrary, the reconstruction of irregularly (or non-equally spaced) sampled signals is a topic still under investigation.

Permission to make digital or hard copies of all or part of this work for personal or classroom use is granted without fee provided that copies are not made or distributed for profit or commercial advantage and that copies bear this notice and the full citation on the first page. To copy otherwise, to republish, to post on servers or to redistribute to lists, requires prior specific permission and/or a fee.

IPSN'07, April 25-27, 2007, Cambridge, Massachusetts, USA.

Copyright 2007 ACM 978-1-59593-638-7/07/0004 ...\$5.00.
The irregular sampling theory is concerned with the problem of recovering a bandlimited signal from a sequence of samples, which may be taken in an irregular way. Several reconstruction algorithms have been proposed in the literature (see e.g., [1]) and have found application in a variety of fields, such as digital medical imaging [2,3], geophysics [4], weather forecast [5], astronomy, and oceanography [6].

Recently, a great deal of attention has been devoted to sensor networks, whose nodes sample a physical field, like air temperature, light intensity, pollution levels or rain falls, and report the data to a common processing unit ( $\sin k$ node). The sink node is in charge of reconstructing the sensed field. In general, sensors are not regularly deployed in the area of interest and, if not synchronized to a common reference, their sampling time also differ. As a result the physical field is not equally-spaced sampled both in the space and in the time domain. If the field is bandlimited in both domains, then an estimate of the discrete spectrum can be provided, although high sampling irregularities typically result in a degradation of the reconstructed signal. The work in [7] investigates this issue showing how irregular spatio-temporal sampling affects the performance in sensor networks. Other interesting studies can be found in [8] and [9], just to name a few, which address the perturbations of regular sampling in shift-invariant spaces [8] and the reconstruction of irregularly sampled images in presence of measure noise [9]. Some conditions on the irregular topology of a sensor network, which allow for a successful signal reconstruction, are identified in [10]. There, however, it is assumed that the reconstruction algorithm has perfect knowledge of the sensors position and measures are noise free: the failure in reconstruction is only due to the finite machine precision. Such assumptions are removed in [11] where sensors are uniformly distributed over the spatial observation interval and may move around a known average location; the effects of noisy measures and jittered positions are analyzed when linear reconstruction techniques are employed.

Similarly to $[10,11]$, in this work we consider wireless sensor networks and investigate a reconstruction technique that follows the work by Feichtinger [1]. Such a technique does not require interpolation and can be viewed as a generalization of the classical discrete Fourier transform (DFT) to irregularly sampled signals. Although the theory applies to fields in any dimension, for simplicity, we study the unidimensional case in the space domain, i.e., we assume that sensors are deployed irregularly on a finite spatial interval. As discussed later, the extension to multidimensional space-time fields is straightforward. We consider that the field samples are corrupted by additive noise, due to quantization, round-off errors or quality of the sensing devices. Moreover, the sink does not have perfect knowledge of the sensor positions, as is the case when they are estimated through a localization technique [12-14]. 
Notice that, differently from our previous work in [11], here sensors are fixed and their position is known at the sink with some error. Furthermore, our goal in this work is to evaluate the performance of the considered reconstruction technique in the presence of quasi-equally spaced sensor layouts. The motivation for considering such network topologies is that typically terrain conditions and deployment practicality make regular sensors placement unfeasible. For the sake of comparison, we also study the case of sensors randomly distributed in the network area with a uniform distribution. Through asymptotic analysis, we derive the mean square error (MSE) on the reconstructed signal, as a function of the network characteristics. Different network topologies are considered and compared; in particular, for two of them, under some assumptions on matrix freeness we find an approximate closed form expression of the MSE, which proved to be very tight.

We highlight that our results play a key role in the design of sensor networks and that, to the best of our knowledge, no previous work has analytically studied the impact of irregular sensor layouts on field reconstruction.

The rest of the paper is organized as follows. Section 2 introduces the system model, Section 3 defines our performance metric and gives some details on the field reconstruction technique. In Section 4 we present the asymptotic analysis of the system performance, and validate the analytical results through numerical simulations. Finally, Section 5 concludes the paper.

\section{SYSTEM MODEL}

A unidimensional bandlimited physical field, described by $2 M+$ 1 harmonics, can be written as

$$
s(x)=\frac{1}{\sqrt{2 M+1}} \sum_{k=-M}^{M} a_{k} \mathrm{e}^{-\mathrm{j} 2 \pi k x}
$$

The field is sampled in the space domain by $r$ sensors, which are deployed in the normalized interval $[-1 / 2,1 / 2)$. The sensor positions are given by the vector $\mathrm{x}=\left[x_{1}, \ldots, x_{r}\right]^{\mathrm{T}}, x_{q} \in[-1 / 2,1 / 2)$, $q=1, \ldots, r$. Then, let $\mathbf{s}=\left[s\left(x_{1}\right), \ldots, s\left(x_{r}\right)\right]^{\mathrm{T}}$. The discrete spectrum of $s(x)$ is denoted by the random column vector $\mathrm{a}=$ $\left[a_{-M}, \ldots, a_{+M}\right]^{\mathrm{T}}$ and we assume $\mathbb{E}\left[\|\mathbf{a}\|^{2}\right]=\sigma_{a}^{2} \mathbf{I}^{1}$.

We define $\beta$ as the ratio of the number of complex harmonics that describe the field to the number of sensors:

$$
\beta=\frac{2 M+1}{r}
$$

Notice that for equally spaced sensors the sampling theorem is satisfied for $\beta \leq 1$, we therefore focus our analysis on this range of values.

Following [1], the values of the field at positions $\mathrm{x}$ are given by the size $r$ column vector

$$
\mathbf{s}=\mathbf{G}_{\mathbf{x}}^{\dagger} \mathbf{a}
$$

where $(\cdot)^{\dagger}$ is the conjugate transpose operator and $\mathbf{G}_{\mathbf{x}}$ is the $(2 M+$ 1) $\times r$ matrix defined as:

$$
\begin{aligned}
\left(\mathbf{G}_{\mathbf{x}}\right)_{k q} & =\frac{1}{\sqrt{2 M+1}} \mathrm{e}^{-\mathrm{j} 2 \pi k x_{q}} \\
& =\sqrt{\frac{\beta}{r}} \mathrm{e}^{-\mathrm{j} 2 \pi k x_{q}}
\end{aligned}
$$

for $q=1, \ldots, r$ and $k=-M, \ldots,+M$. Notice that, if the positions $x_{q}$ are equally spaced (i.e., $\left.x_{q}=(q-1 / 2) / r-1 / 2\right)$, then $\sqrt{\beta} \mathbf{G}_{\mathbf{x}}$ is unitary (i.e., $\beta \mathbf{G}_{\mathbf{x}} \mathbf{G}_{\mathbf{x}}^{\dagger}=\mathbf{I}$ ) [15]. It can be shown that,

\footnotetext{
${ }^{1} \mathbb{E}[\cdot]$ is the average operator and the identity matrix is denoted by $\mathbf{I}$
}

in the multidimensional case, the relation between field spectrum and samples is similar to (1), with a different structure of the matrix $\mathbf{G}_{\mathbf{x}}$. We therefore limit our investigation to the unidimensional case, since the extension to multidimensional fields is straightforward and considerations similar to the unidimensional case can be drawn.

When the measures provided by sensors are affected by noise, we can write:

$$
\mathbf{p}=\mathbf{s}+\mathbf{n}=\mathbf{G}_{\mathbf{x}}^{\dagger} \mathbf{a}+\mathbf{n}
$$

where the noise is represented by the $r$-size, zero-mean random vector $\mathbf{n}$, with covariance matrix $\mathbb{E}\left[\mathbf{n} \mathbf{n}^{\dagger}\right]=\sigma_{n}^{2} \mathbf{I}_{2 M+1}$. Let us define:

$$
\mathrm{SNR}_{m}=\frac{\sigma_{a}^{2}}{\sigma_{n}^{2}}=\frac{1}{\alpha}
$$

as the signal-to-noise ratio on the measure.

Measures are sent to the so-called sink node whose task is to provide an accurate field reconstruction; data transmissions between nodes and sink are considered to be error free. However, we assume that the sink does not have perfect knowledge of the network topology and that an estimate of the sensors position is given by:

$$
\hat{\mathbf{x}}=\mathbf{x}+\boldsymbol{\delta}
$$

where the vector $\delta$ represents the position error. The entries of $\delta$ are modeled as independent and identically distributed, zero-mean random variables with probability density function $f_{\delta}(x)$, variance $\sigma_{\delta}^{2}$, and covariance matrix $\mathbb{E}\left[\boldsymbol{\delta} \delta^{\dagger}\right]=\sigma_{\delta}^{2} \mathbf{I}_{2 M+1}$. The random vectors $\delta, \mathrm{n}$, and a are assumed to be uncorrelated.

Both the noise $\mathbf{n}$ and the position error $\boldsymbol{\delta}$ affect the quality of the reconstructed field. In practice, high reconstruction quality is expected when the standard deviation $\sigma_{\delta}$ is small compared to the average sensor separation. Since $r$ sensors are deployed in the interval $[-1 / 2,1 / 2)$, the average sensor separation is $1 / r$. We therefore define the parameter

$$
\rho=\frac{\sigma_{\delta}}{1 / r}
$$

as a measure of the uncertainty on the network topology at the sink; when $\rho=0$, we have perfect knowledge of the topology.

\section{PERFORMANCE METRIC AND RECON- STRUCTION TECHNIQUES}

The task of the reconstruction algorithm is to compute an estimate â of the spectrum a. Given â, the estimate $\hat{s}(x)$ of the field $s(x)$ can be obtained as:

$$
\hat{s}(x)=\frac{1}{\sqrt{2 M+1}} \sum_{k=-M}^{M} \hat{a}_{k} \mathrm{e}^{-\mathrm{j} 2 \pi k x}
$$

As a reconstruction performance metric we consider the MSE of the field estimate which, for any given $\mathbf{x}$ is defined as:

$$
\begin{aligned}
\operatorname{MSE}_{\mathbf{x}} & =\underset{\mathbf{a}, \mathbf{n}, \delta}{\mathbb{E}}\left[\int_{-\frac{1}{2}}^{\frac{1}{2}}|\hat{s}(x)-s(x)|^{2} \mathrm{~d} x\right] \\
& =\frac{1}{2 M+1} \underset{\mathbf{a}, \mathbf{n}, \boldsymbol{\delta}}{\mathbb{E}}\left[\|\hat{\mathbf{a}}-\mathbf{a}\|^{2}\right]
\end{aligned}
$$

where the operator $\mathbb{E}[\cdot]$ averages with respect to the subscripted random vectors.

For linear models such as (3), several estimation techniques based on linear filtering are available in the literature [16]. The idea is to 
employ a suitable filter B such that the estimate of the field spectrum is given by the linear operation

$$
\hat{\mathbf{a}}=\mathbf{B}^{\dagger} \mathbf{p}
$$

where $\mathbf{B}$ is an $r \times(2 M+1)$ matrix. Here we focus on the linear filter providing the best performance in terms of MSE, that is, the linear minimum MSE (LMMSE) filter. In our case this is given by [16]:

$$
\mathbf{B}=\mathbf{G}_{\mathbf{x}}^{\dagger}\left(\mathbf{R}_{\mathbf{x}}+\alpha \mathbf{I}\right)^{-1}
$$

where $\mathbf{R}_{\mathbf{x}}=\mathbf{G}_{\mathbf{x}} \mathbf{G}_{\mathbf{x}}^{\dagger}$ is Toeplitz and Hermitian. Notice that (7) requires the knowledge of $\mathbf{x}$, which is not available at the sink. Therefore we use the only available information and replace $\mathbf{x}$ by its estimate $\hat{\mathbf{x}}$, and employ the filter

$$
\mathbf{B}=\mathbf{G}_{\hat{\mathbf{x}}}^{\dagger}\left(\mathbf{R}_{\hat{\mathbf{x}}}+\alpha \mathbf{I}\right)^{-1}
$$

The matrix $\mathbf{G}_{\hat{\mathbf{x}}}$ is defined as in (2), where $x_{q}$ is replaced by $\hat{x}_{q}$, and $\mathbf{R}_{\hat{\mathbf{x}}}=\mathbf{G}_{\hat{\mathbf{x}}} \mathbf{G}_{\hat{\mathbf{x}}}^{\dagger}$. We further assume that the value of $\mathbf{S N R}_{m}=\alpha^{-1}$ is known. Substituting (6) in (5) and using (3), after some algebra, we write the MSE as:

$$
\begin{aligned}
\operatorname{MSE}_{\mathbf{x}}= & \frac{1}{2 M+1} \underset{\mathbf{a}, \mathbf{n}, \boldsymbol{\delta}}{\mathbb{E}}\left[\left\|\mathbf{B}^{\dagger}\left(\mathbf{G}_{\mathbf{x}} \mathbf{a}+\mathbf{n}\right)-\mathbf{a}\right\|^{2}\right] \\
= & \frac{\sigma_{a}^{2}}{2 M+1} \operatorname{Tr}\left\{\underset{\delta}{\mathbb{E}}\left[\mathbf{G}_{\mathbf{x}} \mathbf{G}_{\hat{\mathbf{x}}}^{\dagger}\left(\mathbf{R}_{\hat{\mathbf{x}}}+\alpha \mathbf{I}\right)^{-2} \mathbf{G}_{\hat{\mathbf{x}}} \mathbf{G}_{\mathbf{x}}^{\dagger}\right]\right. \\
& +\alpha \underset{\delta}{\mathbb{E}}\left[\mathbf{R}_{\hat{\mathbf{x}}}\left(\mathbf{R}_{\hat{\mathbf{x}}}+\alpha \mathbf{I}\right)^{-2}\right]+\mathbf{I} \\
& \left.-2 \Re\left\{\underset{\delta}{\mathbb{E}}\left[\mathbf{G}_{\mathbf{x}} \mathbf{G}_{\hat{\mathbf{x}}}^{\dagger}\left(\mathbf{R}_{\hat{\mathbf{x}}}+\alpha \mathbf{I}\right)^{-1}\right]\right\}\right\}
\end{aligned}
$$

where $\operatorname{Tr}\{\cdot\}$ and $\Re\{\cdot\}$ are the trace and real part operator, respectively. Clearly (9) is a function of a given network topology defined by $x$. In a more general case we consider the topology to be random, with independent positions $x_{q}$ having probability density functions (pdf) $f_{q}(x), q=1, \ldots, r$. In particular, in this work we compare different topology distributions, labeled as follows (see Figure 1):

- Uniform-1: where $x_{q}$ has zero mean and is uniformly distributed in $[-1 / 2,1 / 2)$;

- Uniform-2: where $x_{q}$ has mean $\bar{x}_{q}=(q-1 / 2) / r-1 / 2$, and is uniformly distributed in $\left[\bar{x}_{q}-\frac{1}{2 r}, \bar{x}_{q}+\frac{1}{2 r}\right)$, with variance $\frac{1}{12 r^{2}}$;

- Gaussian: where $x_{q}$ has mean $\bar{x}_{q}=(q-1 / 2) / r-1 / 2$, and is Gaussian distributed with mean $\bar{x}_{q}$ and variance $\sigma_{g}^{2}$. Similarly to (4), it is useful to write $\sigma_{g}^{2}$ in terms of the average sensor separation $1 / r$ through the parameter $\eta$, that is $\sigma_{g}^{2}=$ $\eta^{2} / r^{2}$.

The Uniform- 1 distribution assumes that sensors are deployed with uniform distribution over the entire sampling region. One of the reasons for analyzing this distribution is that, among all others, it has the highest possible variance and, as will be clear in Section 4, provides the worst performance. Instead, Uniform-2 and Gaussian reflect the attempt to deploy sensors in a quasi-equally spaced manner. This is the case when a regular sensors placement is desirable but, due to terrain conditions or deployment practicality, it turns out to be not achievable. Notice that the support of the Gaussian distribution is infinite and, thus, with a non zero probability some of the samples $x_{q}$ fall outside the range $[-1 / 2,1 / 2)$. In principle such values should be discarded. In practice, we consider them as valid sampling points by wrapping them around. Note that, when

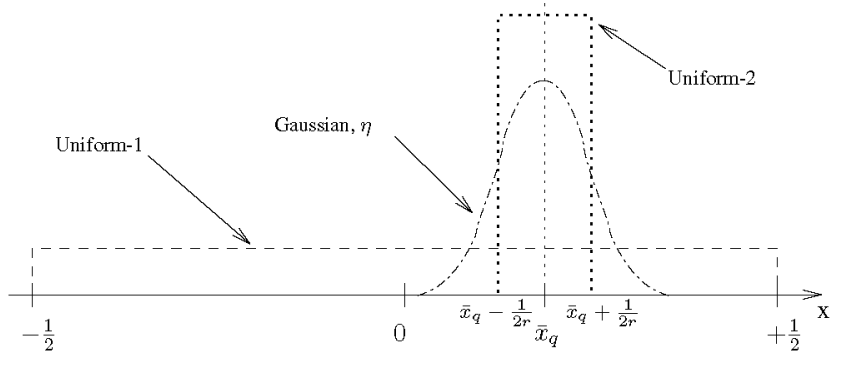

Figure 1: Qualitative representations of the pdfs Uniform-1, Uniform-2 and Gaussian

the distribution variance is small, the effect of such operation is negligible.

In the following the three distributions are compared in terms of the MSE that is obtained on the reconstructed field.

\section{ASYMPTOTIC ANALYSIS OF THE MSE}

A simple and effective tool to evaluate the performance of large finite systems is asymptotic analysis. In our case, we let the field number of harmonics $2 M+1$ and the number of samples $r$ grow to infinity, while the ratio $\beta=(2 M+1) / r$ is kept constant. The results show the validity of our asymptotic analysis, even for small values of $M$ and $r$.

To carry out our asymptotic analysis, we consider as performance metric the asymptotic per-sample average MSE, normalized to $\sigma_{a}^{2}$ :

$$
\mathrm{MSE}_{\infty}=\lim _{\substack{, r \rightarrow \\ \beta}} \frac{1}{\sigma_{a}^{2}} \underset{\mathbf{x}}{\mathbb{E}}\left[\mathrm{MSE}_{\mathbf{x}}\right]
$$

Also, for simplicity of notation we introduce the linear functional [17]

$$
\phi(\mathbf{X})=\lim _{n \rightarrow+\infty} \frac{1}{n} \operatorname{Tr}\{\mathbb{E}[\mathbf{X}]\}
$$

where the argument $\mathbf{X}$ is an $n \times n$ random square matrix. The functional $\phi(\cdot)$ has many interesting properties; here we report those that will be useful in the following analysis:

$$
\phi(\mathbf{I})=1, \quad \phi(g(\mathbf{X}))=\mathbb{E}[g(\xi)],
$$

$$
\phi\left(\mathbf{X}_{1}+\mathbf{X}_{2}\right)=\phi\left(\mathbf{X}_{1}\right)+\phi\left(\mathbf{X}_{2}\right), \quad \phi(a \mathbf{X})=\phi(a) \phi(\mathbf{X})
$$

where $\xi$ is the random variable with the asymptotic eigenvalue distribution of $\mathbf{X}, g(\cdot)$ is an analytic function ${ }^{2}$, and $a$ is a scalar (see [17] for further details). From (9) and using the above definitions, we obtain

$$
\begin{aligned}
& \operatorname{MSE}_{\infty}=\phi\left(\mathbf{G}_{\mathbf{x}} \mathbf{G}_{\hat{\mathbf{x}}}^{\dagger}\left(\mathbf{R}_{\hat{\mathbf{x}}}+\alpha \mathbf{I}\right)^{-2} \mathbf{G}_{\hat{\mathbf{x}}} \mathbf{G}_{\mathbf{x}}^{\dagger}\right)+1 \\
& \quad+\alpha \phi\left(\mathbf{R}_{\hat{\mathbf{x}}}\left(\mathbf{R}_{\hat{\mathbf{x}}}+\alpha \mathbf{I}\right)^{-2}\right)-2 \Re \phi\left(\mathbf{G}_{\mathbf{x}} \mathbf{G}_{\hat{\mathbf{x}}}^{\dagger}\left(\mathbf{R}_{\hat{\mathbf{x}}}+\alpha \mathbf{I}\right)^{-1}\right)
\end{aligned}
$$

\subsection{Uniform-1 topology}

Clearly (10) depends on the distribution of $\mathbf{x}$. We first analyze the Uniform- 1 distribution, i.e., the case where $x_{q} \sim \mathcal{U}[-1 / 2,+1 / 2)$.

\footnotetext{
${ }^{2}$ Note the small abuse of notation when using $g(\cdot)$ for both scalar and matrix argument
} 
Then, the following results hold:

$$
\begin{aligned}
\phi\left(\mathbf{R}_{\hat{\mathbf{x}}}^{p}\right) & =\phi\left(\mathbf{R}_{\mathbf{x}}^{p}\right) \\
\phi\left(\mathbf{G}_{\mathbf{x}} \mathbf{G}_{\hat{\mathbf{x}}}^{\dagger} \mathbf{R}_{\hat{\mathbf{x}}}^{p}\right) & =\phi\left(\mathbf{G}_{\hat{\mathbf{x}}} \mathbf{G}_{\mathbf{x}}^{\dagger} \mathbf{R}_{\mathbf{x}}^{p}\right) \\
\phi\left(\mathbf{G}_{\mathbf{x}} \mathbf{G}_{\hat{\mathbf{x}}}^{\dagger} \mathbf{R}_{\hat{\mathbf{x}}}^{p} \mathbf{G}_{\hat{\mathbf{x}}} \mathbf{G}_{\mathbf{x}}^{\dagger}\right) & =\phi\left(\mathbf{G}_{\hat{\mathbf{x}}} \mathbf{G}_{\mathbf{x}}^{\dagger} \mathbf{R}_{\mathbf{x}}^{p} \mathbf{G}_{\mathbf{x}} \mathbf{G}_{\hat{\mathbf{x}}}^{\dagger}\right)
\end{aligned}
$$

where $p$ is a positive integer (the proofs are given in Appendix A). Moreover in Appendix B it is shown that:

$$
\begin{aligned}
\underset{\delta}{\mathbb{E}}\left[\mathbf{G}_{\hat{\mathbf{x}}}\right] & =\mathbf{C G}_{\mathbf{x}} \\
\underset{\delta}{\mathbb{E}}\left[\mathbf{G}_{\hat{\mathbf{x}}}^{\dagger} \mathbf{G}_{\hat{\mathbf{x}}}\right] & =\mathbf{G}_{\mathbf{x}}^{\dagger} \mathbf{C}^{\dagger} \mathbf{C G}_{\mathbf{x}}+\nu \mathbf{I}
\end{aligned}
$$

where $\nu=1-\operatorname{Tr}\left\{\mathbf{C}^{\dagger} \mathbf{C}\right\} /(2 M+1)$ and the $(2 M+1) \times(2 M+1)$ diagonal matrix $\mathrm{C}$ has entries given by:

$$
(\mathbf{C})_{k k}=C_{\delta}(-\mathbf{j} 2 \pi k)
$$

for $k=-M, \ldots, M$, and with $C_{\delta}(s)=\int_{-\infty}^{+\infty} \exp (s x) f_{\delta}(x) \mathrm{d} x$ being the characteristic function of the random variable $\delta$.

From (11) it follows that, if the function $g\left(\mathbf{R}_{\hat{\mathbf{x}}}\right)$ admits a power series in the variable $\mathbf{R}_{\hat{\mathbf{x}}}$ (i.e., $g\left(\mathbf{R}_{\hat{\mathbf{x}}}\right)=\sum_{i=0}^{\infty} c_{i} \mathbf{R}_{\hat{\mathbf{x}}}^{i}$ ), then, thanks to the linearity of the functional $\phi(\cdot)$, we have:

$$
\phi\left(g\left(\mathbf{R}_{\hat{\mathbf{x}}}\right)\right)=\phi\left(g\left(\mathbf{R}_{\mathbf{x}}\right)\right)
$$

. By defining $g\left(\mathbf{R}_{\hat{\mathbf{x}}}\right)=\mathbf{R}_{\hat{\mathbf{x}}}\left(\mathbf{R}_{\hat{\mathbf{x}}}+\alpha \mathbf{I}\right)^{-2}$, the second term of (10) simplifies to $\alpha \phi\left(\mathbf{R}_{\mathbf{x}}\left(\mathbf{R}_{\mathbf{x}}+\alpha \mathbf{I}\right)^{-2}\right)$. Similarly, for the first and fourth term of (10) we obtain, respectively,

$$
\phi\left(\mathbf{G}_{\mathbf{x}} \mathbf{G}_{\hat{\mathbf{x}}}^{\dagger} g\left(\mathbf{R}_{\hat{\mathbf{x}}}\right) \mathbf{G}_{\hat{\mathbf{x}}} \mathbf{G}_{\mathbf{x}}^{\dagger}\right)=\phi\left(\mathbf{G}_{\hat{\mathbf{x}}} \mathbf{G}_{\mathbf{x}}^{\dagger} g\left(\mathbf{R}_{\mathbf{x}}\right) \mathbf{G}_{\mathbf{x}} \mathbf{G}_{\hat{\mathbf{x}}}^{\dagger}\right)
$$

and

$$
\phi\left(\mathbf{G}_{\mathbf{x}} \mathbf{G}_{\hat{\mathbf{x}}}^{\dagger} g\left(\mathbf{R}_{\hat{\mathbf{x}}}\right)\right)=\phi\left(\mathbf{G}_{\hat{\mathbf{x}}} \mathbf{G}_{\mathbf{x}}^{\dagger} g\left(\mathbf{R}_{\mathbf{x}}\right)\right)
$$

Then, the asymptotic MSE reduces to

$$
\begin{aligned}
& \mathrm{MSE}_{\infty}=\phi\left(\mathbf{G}_{\hat{\mathbf{x}}} \mathbf{G}_{\mathbf{x}}^{\dagger}\left(\mathbf{R}_{\mathbf{x}}+\alpha \mathbf{I}\right)^{-2} \mathbf{G}_{\mathbf{x}} \mathbf{G}_{\hat{\mathbf{x}}}^{\dagger}\right)+1 \\
& \quad+\alpha \phi\left(\mathbf{R}_{\mathbf{x}}\left(\mathbf{R}_{\mathbf{x}}+\alpha \mathbf{I}\right)^{-2}\right)-2 \Re \phi\left(\mathbf{G}_{\hat{\mathbf{x}}} \mathbf{G}_{\mathbf{x}}^{\dagger}\left(\mathbf{R}_{\mathbf{x}}+\alpha \mathbf{I}\right)^{-1}\right)
\end{aligned}
$$

Applying the definition of $\phi(\cdot)$, the properties of the trace operator and (15) to the first term of (16), we have:

$$
\begin{aligned}
\phi\left(\mathbf{G}_{\hat{\mathbf{x}}} \mathbf{G}_{\mathbf{x}}^{\dagger}\left(\mathbf{R}_{\mathbf{x}}+\alpha \mathbf{I}\right)^{-2} \mathbf{G}_{\mathbf{x}} \mathbf{G}_{\hat{\mathbf{x}}}^{\dagger}\right) \\
=\phi\left(\mathbf{G}_{\mathbf{x}} \mathbf{G}_{\hat{\mathbf{x}}}^{\dagger} \mathbf{G}_{\hat{\mathbf{x}}} \mathbf{G}_{\mathbf{x}}^{\dagger}\left(\mathbf{R}_{\mathbf{x}}+\alpha \mathbf{I}\right)^{-2}\right) \\
=\phi\left(\mathbf{G}_{\mathbf{x}} \underset{\boldsymbol{\delta}}{\mathbb{E}}\left[\mathbf{G}_{\hat{\mathbf{x}}}^{\dagger} \mathbf{G}_{\hat{\mathbf{x}}}\right] \mathbf{G}_{\mathbf{x}}^{\dagger}\left(\mathbf{R}_{\mathbf{x}}+\alpha \mathbf{I}\right)^{-2}\right) \\
=\phi\left(\mathbf{G}_{\mathbf{x}}\left(\mathbf{G}_{\mathbf{x}}^{\dagger} \mathbf{C}^{\dagger} \mathbf{C G}_{\mathbf{x}}+\nu \mathbf{I}\right) \mathbf{G}_{\mathbf{x}}^{\dagger}\left(\mathbf{R}_{\mathbf{x}}+\alpha \mathbf{I}\right)^{-2}\right) \\
=\phi\left(\mathbf{C}^{\dagger} \mathbf{C} \mathbf{R}_{\mathbf{x}}\left(\mathbf{R}_{\mathbf{x}}+\alpha \mathbf{I}\right)^{-2} \mathbf{R}_{\mathbf{x}}\right) \\
\quad \quad \quad\left(1-\phi\left(\mathbf{C}^{\dagger} \mathbf{C}\right)\right) \phi\left(\mathbf{R}_{\mathbf{x}}\left(\mathbf{R}_{\mathbf{x}}+\alpha \mathbf{I}\right)^{-2}\right)
\end{aligned}
$$

Using (15), the fourth term of (16) can be rewritten as:

$$
\begin{aligned}
\phi\left(\mathbf{G}_{\hat{\mathbf{x}}} \mathbf{G}_{\mathbf{x}}^{\dagger}\left(\mathbf{R}_{\mathbf{x}}+\alpha \mathbf{I}\right)^{-1}\right) & =\phi\left(\underset{\delta}{\mathbb{\delta}}\left[\mathbf{G}_{\hat{\mathbf{x}}}\right] \mathbf{G}_{\mathbf{x}}^{\dagger}\left(\mathbf{R}_{\mathbf{x}}+\alpha \mathbf{I}\right)^{-1}\right) \\
& =\phi\left(\mathbf{C R}_{\mathbf{x}}\left(\mathbf{R}_{\mathbf{x}}+\alpha \mathbf{I}\right)^{-1}\right)
\end{aligned}
$$

Let us assume that $\mathbf{C}$ and $\mathbf{R}_{\mathbf{x}}$ are asymptotically free matrices [17] (later in the paper, we will show that this is a fair assumption, given the excellent match between analytical and simulation results $)$. Then, $\phi\left(\mathbf{C}^{p_{1}} \mathbf{R}_{\mathbf{x}}^{p_{2}}\right)=\phi\left(\mathbf{C}^{p_{1}}\right) \phi\left(\mathbf{R}_{\mathbf{x}}^{p_{2}}\right)$ for any positive integers $p_{1}, p_{2}$, and the final expression of the asymptotic MSE becomes:

$$
\begin{aligned}
& \operatorname{MSE}_{\infty}=\phi\left(\mathbf{C}^{\dagger} \mathbf{C}\right) \mathbb{E} {\left[\frac{\lambda^{2}}{(\lambda+\alpha \beta)^{2}}\right] } \\
&+\beta\left(1+\alpha-\phi\left(\mathbf{C}^{\dagger} \mathbf{C}\right)\right) \mathbb{E}\left[\frac{\lambda}{(\lambda+\alpha \beta)^{2}}\right] \\
&+1-2 \phi(\Re\{\mathbf{C}\}) \mathbb{E}\left[\frac{\lambda}{\lambda+\alpha \beta}\right]
\end{aligned}
$$

where $\lambda$ is a random variable with pdf $f_{\lambda}(x, \beta)$, distributed as the asymptotic eigenvalues of $\beta \mathbf{R}_{\mathbf{x}}$. Looking at (17), we make the following remarks.

(i) The distribution of $\lambda$ depends on the parameter $\beta$, since $\beta$ appears in the definition of $\mathbf{R}_{\mathbf{x}}$. Thus, in the analysis of the MSE the function $f_{\lambda}(x, \beta)$ completely defines the topology distribution.

(ii) Equation (17) shows that the effects on the MSE induced by the random topology distribution $f_{\lambda}(x, \beta)$ and by the relative noise level $\alpha$ can be decoupled from the effects due to imperfect knowledge of the network topology at the sink (represented by the matrix $\mathbf{C}$ ).

(iii) For the evaluation of the averages in (17) a closed form expression of $f_{\lambda}(x, \beta)$ would be required. To the best of our knowledge such expression is still unknown, although [10] provides an algorithm to evaluate the moments of $\lambda$ in closed form. We compute the averages in (17) numerically.

Figure 2 shows the histograms of $f_{\lambda}(x, \beta)$ for the Uniform-1 topology distribution and different values of $\beta$. Observe that, for $\beta=0(r \rightarrow \infty$, while keeping $M$ constant $)$, the matrix $\mathbf{R}_{\mathbf{x}}$ tends to the identity matrix and, thus, $f_{\lambda}(x, 0)=\delta(x-1)$ with $\delta(\cdot)$ being the Dirac's delta.

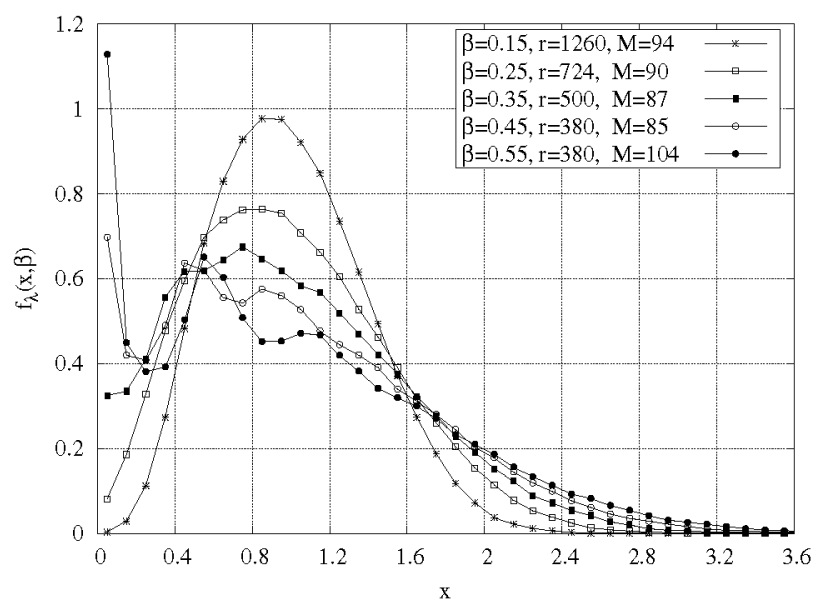

Figure 2: Histograms of $f_{\lambda}(x, \beta)$ for different values of $\beta$

As for the matrix $\mathbf{C}$ we observe that, when the error vector $\boldsymbol{\delta}$ is Gaussian distributed with zero mean and variance $\sigma_{\delta}^{2}$, its characteristic function, $C_{\delta}(s)$, is given by:

$$
C_{\delta}(s)=\exp \left(\sigma_{\delta}^{2} s^{2} / 2\right)
$$


In this case the matrix $\mathbf{C}$ is real and, for a constant $\rho=\sigma_{\delta} r$ and any integer $p$, we have:

$$
\begin{aligned}
\phi\left(\mathbf{C}^{p}\right) & =\lim _{\substack{\beta, r \rightarrow+\infty \\
\beta}} \frac{1}{2 M+1} \operatorname{Tr}\left\{\mathbf{C}^{p}\right\} \\
& =\lim _{M, r \rightarrow+\infty} \frac{1}{2 M+1} \sum_{k=-M}^{+M}\left(C_{\delta}(-\mathbf{j} 2 \pi k)\right)^{p} \\
& =\lim _{M, r \rightarrow+\infty} \frac{1}{2 M+1} \sum_{k=-M}^{+M} \exp \left(-2 \pi^{2} p \sigma_{\delta}^{2} k^{2}\right) \\
& =\lim _{M, r \rightarrow+\infty} \frac{1}{2 M+1} \sum_{k=-M}^{+M} \exp \left(-2 \pi^{2} p \frac{\rho^{2} k^{2}}{r^{2}}\right) \\
& =\int_{-1 / 2}^{+1 / 2} \exp \left(-2 \pi^{2} p \rho^{2} \beta^{2} z^{2}\right) \mathrm{d} z \\
& =\frac{1}{\sqrt{2 \pi \beta^{2} p^{2} p}} \operatorname{erf}\left(\pi \beta \rho \sqrt{\frac{p}{2}}\right)
\end{aligned}
$$

In particular, for $\rho \rightarrow+\infty$ (no knowledge of the network topology) $\phi\left(\mathbf{C}^{p}\right)=0$, while for $\rho=0$ (perfect knowledge of the topology) $\phi\left(\mathbf{C}^{p}\right)=1$. Also, we have: $\lim _{\beta \rightarrow 0} \phi\left(\mathbf{C}^{p}\right)=1$, for any $p$.

Figure 3 shows the MSE obtained when the network has a Uniform1 topology distribution and the LMMSE filter (8) is employed. A Gaussian distribution of the position error $\delta$ is assumed. The solid curves were obtained by computing (17) and averaging over the eigenvalues of 200 realizations of the matrix $\mathbf{R}_{\mathbf{x}}$, with $\beta=0.2$ and $M=200$. The markers represent the average MSE obtained by computing (5) and averaging over 200 realizations of the measures $\mathrm{p}$, with $\beta=0.2$ and $M=20$. The curves are parameterized by $\rho$. The figure also shows the asymptotic results $\rho=0$ (lower bound); the corresponding expressions of the $\mathrm{MSE}_{\infty}$ is given by:

$$
\lim _{\rho \rightarrow 0} \operatorname{MSE}_{\infty}=\mathbb{E}\left[\frac{\alpha \beta}{\lambda+\alpha \beta}\right]
$$

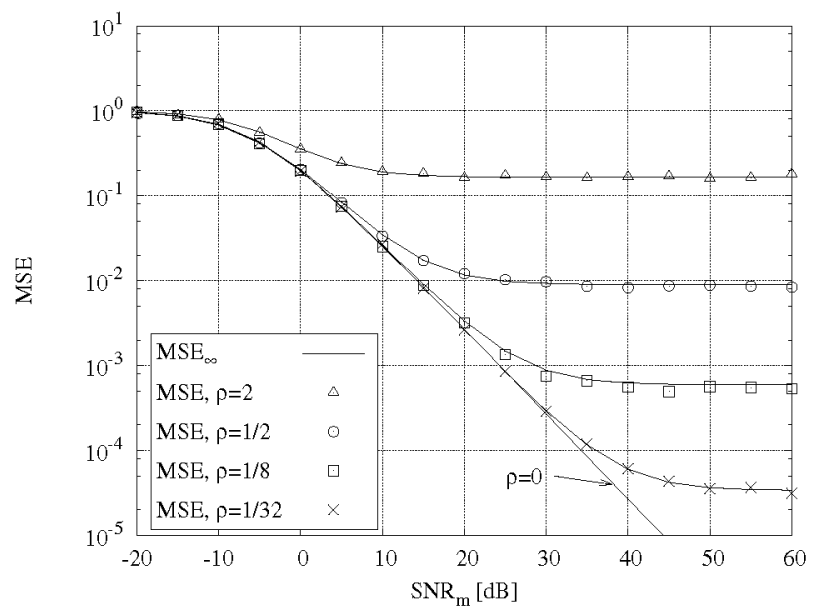

Figure 3: Uniform-1 distribution: $\mathrm{MSE}_{\infty}$ and MSE as a function of $\mathbf{S N R}_{m}(\mathbf{d B})$ and parameterized by $\rho$, with $\beta=0.2$

Looking at the plot in Figure 3, we observe that, as expected, a better knowledge of the network topology results in a lower MSE. Furthermore, the tight match between asymptotical results and finite system simulation, even for small values of $M$ and $r$, confirms the validity of the asymptotic analysis as well as the correctness of our assumptions about freeness. As a last remark, we mention that similar results have been obtained for any value of $\beta$.

\subsection{Uniform-2 and Gaussian topologies}

For distributions other than Uniform-1, equalities (11), (12), and (13) do not hold anymore. As an example, below we provide the closed form expression of $\phi\left(\mathbf{R}_{\hat{\mathbf{x}}}^{2}\right)$. When $\mathrm{x}$ follows the Uniform1 distribution and the error vector $\delta$ is Gaussian distributed, we have [10]:

$$
\phi\left(\mathbf{R}_{\hat{\mathbf{x}}}^{2}\right)=\phi\left(\mathbf{R}_{\mathbf{x}}^{2}\right)=\frac{1+\beta}{\beta^{2}}
$$

When $\mathrm{x}$ follows the Uniform-2 distribution, we have:

$$
\begin{aligned}
\phi\left(\mathbf{R}_{\hat{\mathbf{x}}}^{2}\right) & =\frac{1+\beta}{\beta^{2}} \\
& -\frac{1}{\beta} \iint_{\mathcal{A}} \operatorname{sinc}^{2}\left(\beta z_{1}-\beta z_{2}\right) \mathrm{e}^{\left(-4 \pi^{2} \rho^{2} \beta^{2}\left(z_{1}-z_{2}\right)^{2}\right)} \mathrm{d} z_{1} \mathrm{~d} z_{2}
\end{aligned}
$$

and

$$
\phi\left(\mathbf{R}_{\mathbf{x}}^{2}\right)=\frac{1+\beta}{\beta^{2}}-\frac{1}{\beta} \iint_{\mathcal{A}} \operatorname{sinc}^{2}\left(\beta z_{1}-\beta z_{2}\right) \mathrm{d} z_{1} \mathrm{~d} z_{2}
$$

while, when the entries of $\mathbf{x}$ follow the Gaussian distribution, we obtain:

$$
\begin{aligned}
\phi\left(\mathbf{R}_{\tilde{\mathbf{x}}}^{2}\right) & =\frac{1+\beta}{\beta^{2}} \\
& -\frac{1}{\beta} \iint_{\mathcal{A}} \mathrm{e}^{\left(-4 \pi^{2} \eta^{2} \beta^{2}\left(z_{1}-z_{2}\right)^{2}\right)} \mathrm{e}^{\left(-4 \pi^{2} \beta^{2} \rho^{2}\left(z_{1}-z_{2}\right)^{2}\right)} \mathrm{d} z_{1} \mathrm{~d} z_{2}
\end{aligned}
$$

and

$$
\phi\left(\mathbf{R}_{\mathbf{x}}^{2}\right)=\frac{1+\beta}{\beta^{2}}-\frac{1}{\beta} \iint_{\mathcal{A}} \mathrm{e}^{\left(-4 \pi^{2} \eta^{2} \beta^{2}\left(z_{1}-z_{2}\right)^{2}\right)} \mathrm{d} z_{1} \mathrm{~d} z_{2}
$$

where the set $\mathcal{A} \in \mathbb{R}^{2}$ is defined as

$$
\mathcal{A}=\left\{\left(z_{1}, z_{2}\right) \mid z_{1} \in\left[-\frac{1}{2}, \frac{1}{2}\right), z_{2} \in\left[-\frac{1}{2}, \frac{1}{2}\right), z_{1} \neq z_{2}\right\}
$$

The proof is omitted but can be easily obtained using the definitions of $\phi(\cdot)$ and $\mathbf{R}_{\hat{\mathbf{x}}}$. Notice that the approximation $\phi\left(\mathbf{R}_{\hat{\mathbf{x}}}^{2}\right) \approx$ $\phi\left(\mathbf{R}_{\mathbf{x}}^{2}\right)$ is tight when $\exp \left(-4 \pi^{2} \beta^{2} \rho^{2}\left(x_{1}-x_{2}\right)^{2}\right) \approx 1$, i.e., for $\rho \ll 2 /(\pi \beta)$. Under this condition, we observed that also the approximations:

$$
\begin{aligned}
\phi\left(\mathbf{R}_{\hat{\mathbf{x}}}^{p}\right) & \approx \phi\left(\mathbf{R}_{\mathbf{x}}^{p}\right) \\
\phi\left(\mathbf{G}_{\mathbf{x}} \mathbf{G}_{\hat{\mathbf{x}}}^{\dagger} \mathbf{R}_{\hat{\mathbf{x}}}^{p}\right) & \approx \phi\left(\mathbf{G}_{\hat{\mathbf{x}}} \mathbf{G}_{\mathbf{x}}^{\dagger} \mathbf{R}_{\mathbf{x}}^{p}\right) \\
\phi\left(\mathbf{G}_{\mathbf{x}} \mathbf{G}_{\hat{\mathbf{x}}}^{\dagger} \mathbf{R}_{\hat{\mathbf{x}}}^{p} \mathbf{G}_{\hat{\mathbf{x}}} \mathbf{G}_{\mathbf{x}}^{\dagger}\right) & \approx \phi\left(\mathbf{G}_{\hat{\mathbf{x}}} \mathbf{G}_{\mathbf{x}}^{\dagger} \mathbf{R}_{\mathbf{x}}^{p} \mathbf{G}_{\mathbf{x}} \mathbf{G}_{\hat{\mathbf{x}}}^{\dagger}\right)
\end{aligned}
$$

are tight, we therefore conclude that the asymptotic MSE can be approximated by (17), with the appropriate distribution of $\lambda$.

Figure 4 shows histograms of $f_{\lambda}(x, \beta)$ for $\beta=0.25,0.50,0.70$ when the entries of $\mathbf{x}$ follow the Uniform-2 (solid lines) and Gaussian (dashed lines) distributions. For the Gaussian distribution we set $\eta^{2}=\frac{1}{12}$, so as to obtain the same variance as in the Uniform-2 case (i.e., $\sigma_{g}^{2}=\frac{1}{12 r^{2}}$ ). The histogram of the two distributions of $\lambda$ look very similar: they are concentrated around $x=1$ even for high values of $\beta$. On the contrary, as depicted in Figure 2, the Uniform- 1 distribution shows a significant fraction of eigenvalues close to zero as $\beta$ increases. The reason for such different behavior is that distributions Uniform-2 and Gaussian guarantee a more regular sensor deployment. Recall that a perfectly regular (equally spaced) distribution of sensors results in $\mathbf{R}_{\mathbf{x}}=\mathbf{I} / \beta$ and $f_{\lambda}(x, \beta)=\delta(x-1)$. For example, a realization of $\mathbf{x}$ in the interval $[-1 / 2,1 / 2)$ such that 


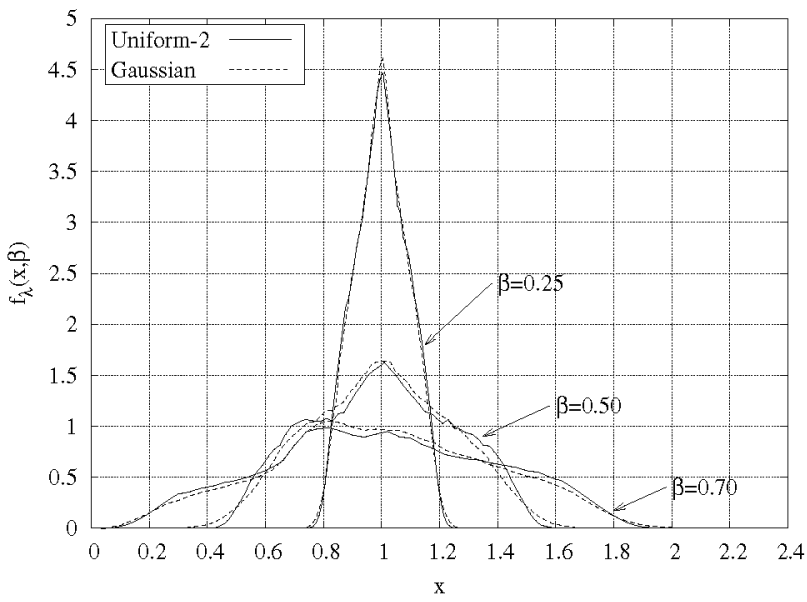

Figure 4: Histograms of $f_{\lambda}(x, \beta)$ for $\beta=0.25,0.50,0.70$, for the Uniform-2 and Gaussian distributions

all $x_{q}<0$, for $q=1, \ldots, r$, is likely to happen for the Uniform-1 distribution, while it is impossible for the Uniform-2 distribution and very unlikely in the case of the Gaussian distribution. Unfortunately, for these network topologies a closed form expression of $f_{\lambda}(x, \beta)$ is unknown and therefore (17) must be computed numerically.

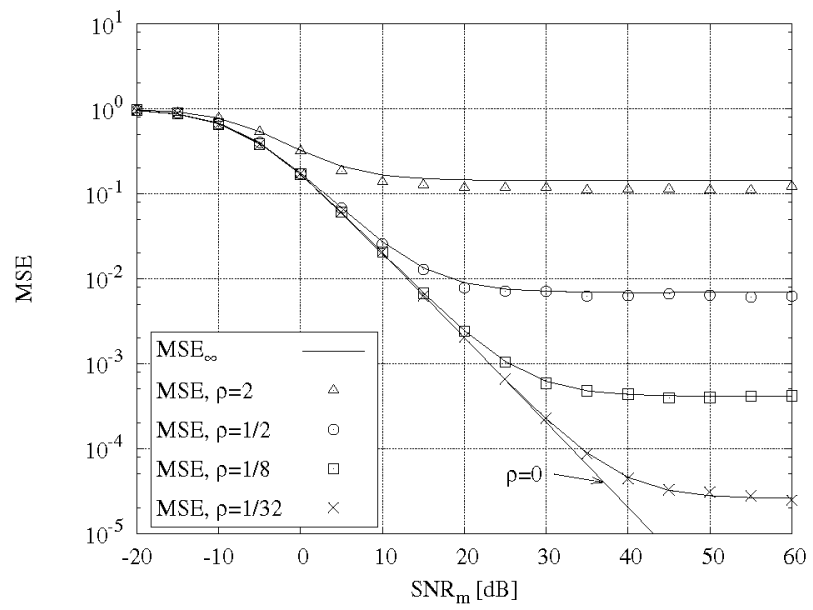

Figure 5: Uniform-2 distribution: $\mathrm{MSE}_{\infty}$ and MSE as a function of $\mathbf{S N R}_{m}$ and for different values of $\rho$

Figure 5 shows the MSE obtained when the network topology follows the Uniform-2 distribution. We used the same parameter setting as in Figure 3. Here the $\mathrm{MSE}_{\infty}$ has been attained through (17), which is an approximation of the true asymptotic MSE; however, the match between the numerical evaluation of the MSE and the results computed through (17) is very tight. Recall that the condition that guarantees a tight approximation is $\rho \ll 2 /(\pi \beta)$; in our case: $2 /(\pi \beta) \approx 3.18$.

Figure 6 compares the $\mathrm{MSE}_{\infty}$ achieved by the Uniform-1 and Uniform-2 distributions, for $\rho=1 / 10$ and different values of $\beta$. The curves have been computed using (17) for both distributions, since the condition $\rho=1 / 10 \ll 2 /(\pi \beta)$ is satisfied. In general, for any given $\beta$, the Uniform-2 distribution provides a lower

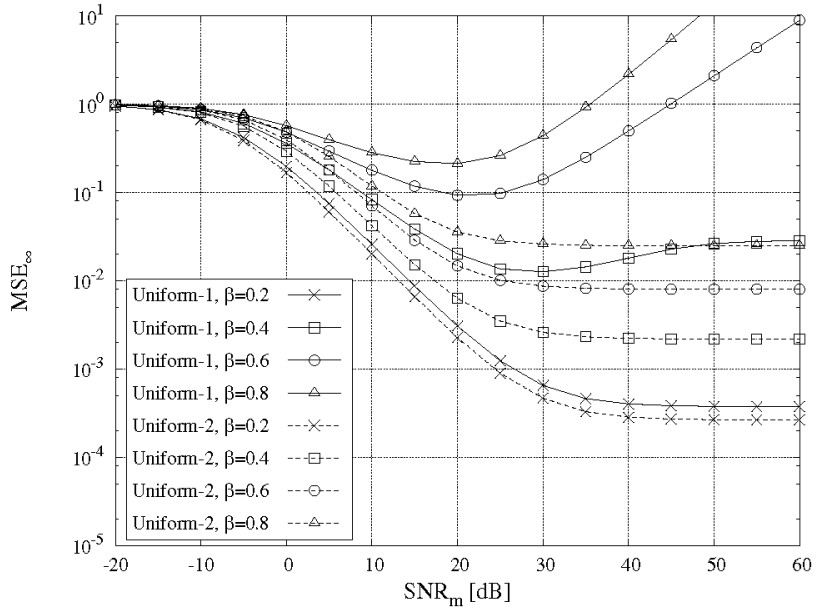

Figure 6: $\mathrm{MSE}_{\infty}$ achieved by the Uniform-1 and Uniform-2 distributions for $\beta=0.2,0.4,0.6,0.8$ and $\rho=1 / 10$

$\mathrm{MSE}_{\infty}$, especially for high $\mathrm{SNR}_{m}$. For high values of $\beta$, the $\mathrm{MSE}_{\infty}$ of the Uniform-1 grows unbounded as $\mathrm{SNR}_{m} \rightarrow \infty$. Indeed, using (17) we notice that

$$
\begin{gathered}
\lim _{\alpha \rightarrow 0} \operatorname{MSE}_{\infty}=\phi\left(\mathbf{C}^{\dagger} \mathbf{C}\right)+\left(1-\phi\left(\mathbf{C}^{\dagger} \mathbf{C}\right)\right) \mathbb{E}\left[\frac{\beta}{\lambda}\right] \\
+1-2 \phi(\Re\{\mathbf{C}\})
\end{gathered}
$$

is dominated by the term $\mathbb{E}[1 / \lambda]$, which in [11] has been shown to diverge for $\beta>0.35$.

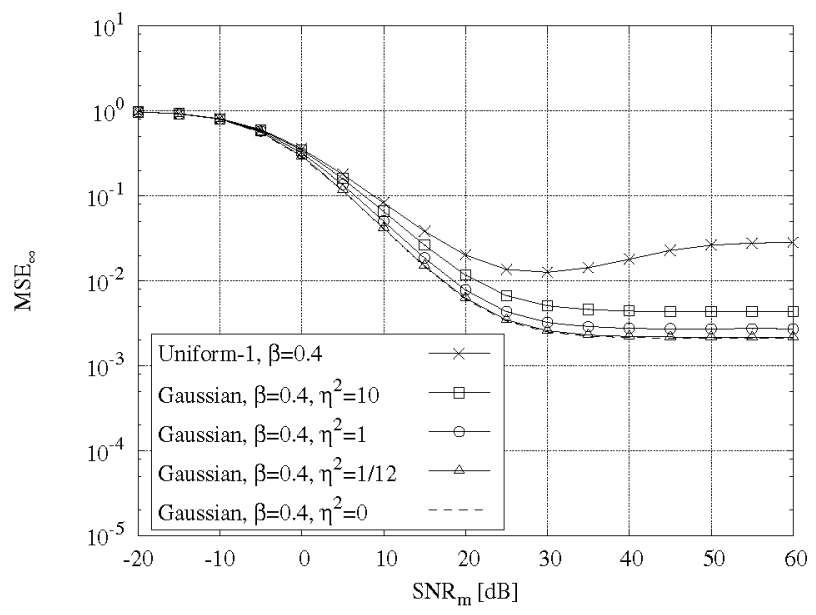

Figure 7: $\mathrm{MSE}_{\infty}$ achieved by the Gaussian topology distribution for $\beta=0.4, \rho=1 / 10$, and $\eta^{2}=0,1 / 12,1,10$

Figure 7 shows the $\mathrm{MSE}_{\infty}$ achieved by the Gaussian topology distribution, for $\beta=0.4, \rho=1 / 10$, and $\eta^{2}=0,1 / 12,1,10$. For the same values of $\beta$ and $\rho$, the Uniform-2 distribution gives very similar results to those obtained for the Gaussian distribution with $\eta^{2}=1 / 12$. As expected, lower values of $\eta^{2}$ result in a more equally spaced sensor deployment and, thus, in a lower $\mathrm{MSE}_{\infty}$. Note that $\eta^{2}=0$ results in a perfectly equally spaced deployment of the sensors. Also, the difference between the performance of the equally spaced deployment $\left(\eta^{2}=0\right)$ and the performance of 
a Gaussian deployment with $\eta^{2}=1 / 12$ is negligible. The above observations hold for any value of $\beta$.

\subsection{Closed form approximation}

To derive a closed form approximation of (17), a closed form approximation of $f_{\lambda}(x, \beta)$ is required. Figure 4 shows that, for the Uniform-2 and Gaussian network topologies, the shape of the empirical pdf of $\lambda$ is well approximated by a triangle with vertices at coordinates $\{(1-a, 0),(1+a, 0),(0,1 / a)\}$, where $0 \leq a \leq 1$ is a parameter. Such triangular distribution is defined by the pdf

$$
f_{t}(x, \beta)= \begin{cases}(x-1+a) / a^{2} & 1-a \leq x \leq 1 \\ (1-x+a) / a^{2} & 1<x \leq 1+a \\ 0 & \text { elsewhere }\end{cases}
$$

which has average $\mu_{t}=1$ and variance $\sigma_{t}^{2}=\frac{a^{2}}{6}$. The condition $a \leq 1$ is required in order to avoid negative eigenvalues. The variance of $\lambda$ is given by

$$
\sigma_{\lambda}^{2}=\mathbb{E}\left[\lambda^{2}\right]-\mathbb{E}[\lambda]^{2}=\phi\left(\beta^{2} \mathbf{R}_{\mathbf{x}}^{2}\right)-\phi\left(\beta \mathbf{R}_{\mathbf{x}}\right)^{2}
$$

Since $\phi\left(\beta \mathbf{R}_{\mathbf{x}}\right)=1$ for any topology distribution, solving (19) and (20), the variance of $\lambda$ can be written as:

$$
\begin{aligned}
\sigma_{\lambda}^{2}=\beta- & \frac{2 \cos ^{2}(\pi \beta)-2+\mathrm{Ci}(2 \pi \beta)-\ln (2 \pi \beta)-\gamma}{\pi^{2} \beta} \\
-\frac{2}{\pi} \operatorname{Si}(2 \pi \beta) &
\end{aligned}
$$

for the Uniform-2, and as

$$
\sigma_{\lambda}^{2}=\beta-\frac{\operatorname{erf}(2 \pi \beta \eta)}{2 \sqrt{\pi} \eta}+\frac{1-\exp \left(-4 \pi^{2} \beta^{2} \eta^{2}\right)}{4 \pi^{2} \beta \eta^{2}}
$$

for the Gaussian distribution. The functions $\mathrm{Ci}(\cdot)$ and $\mathrm{Si}(\cdot)$, and the constant $\gamma$ are the cosine integral, the sine integral and the Euler-Mascheroni constant [18], respectively. For low values of $\beta$, the variance of $\lambda$ can be approximated by $\sigma_{\lambda}^{2} \approx \frac{\pi^{2}}{18} \beta^{3}$ for the Uniform-2 distribution, and by $\sigma_{\lambda}^{2} \approx \frac{2 \pi^{2}}{3} \eta^{2} \beta^{3}$ for the Gaussian distribution.

The parameter $a$ of the triangular pdf can be obtained by setting $\sigma_{t}^{2}=\sigma_{\lambda}^{2}$, i.e.,

$$
a=\sqrt{6 \sigma_{\lambda}^{2}}
$$

Using the triangular distribution, the asymptotic MSE (17) is approximated by

$$
\begin{gathered}
\mathrm{MSE}_{\infty} \approx \phi\left(\mathrm{C}^{\dagger} \mathrm{C}\right) I_{1}+I_{2} \beta\left(1+\alpha-\phi\left(\mathbf{C}^{\dagger} \mathbf{C}\right)\right) \\
+1-2 I_{3} \phi(\Re\{\mathrm{C}\})
\end{gathered}
$$

where the integrals over the support $\Delta$ of the triangular pdf

$$
\begin{aligned}
& I_{1}=\int_{\Delta} \frac{x^{2}}{(x+\alpha \beta)^{2}} f_{t}(x, \beta) \mathrm{d} x \\
& I_{2}=\int_{\Delta} \frac{x}{(x+\alpha \beta)^{2}} f_{t}(x, \beta) \mathrm{d} x \\
& I_{3}=\int_{\Delta} \frac{x}{x+\alpha \beta} f_{t}(x, \beta) \mathrm{d} x
\end{aligned}
$$

can be evaluated in closed form expression.

For the Uniform-2 distribution, Figure 8 compares the $\mathrm{MSE}_{\infty}$ given by the triangular approximation (21) (dashed lines) and the $\mathrm{MSE}_{\infty}$ given by (17) (points), for $\rho=1 / 10$ and $\beta=0.2,0.4,0.6,0.7$. The match is very tight for all values of $\beta$ and all $\mathrm{SNR}_{m} \mathrm{~s}$. The triangular approximation showed to be very tight also in the case of the Gaussian distribution.

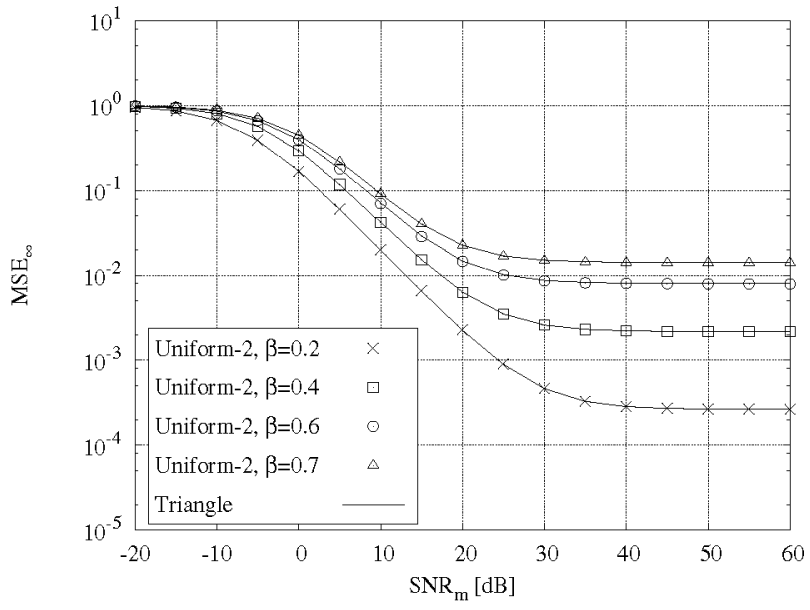

Figure 8: Uniform-2 distribution: $\mathrm{MSE}_{\infty}$ given by (17) and its triangular approximation $(21)$, for $\rho=1 / 10$, and $\beta=$ $0.2,0.4,0.6,0.7$

To conclude, below we give an example of how our results can be used to deploy sensors so as to obtain the desired quality of the reconstructed field.

Example : A network characterized by $\beta=0.4, \rho=1 / 10$, and $S N R_{m}=30 \mathrm{~dB}$ is deployed by an airplane throwing sensors at equally spaced time instants. The unpredictable effects of winds result in a non perfectly equally spaced deployment on the ground. The distribution of the deployment error is assumed to be Gaussian with parameter $\eta$. We want to determine how accurate the sensor deployment need to be, i.e., the maximum $\eta$ for which the network provides an MSE not larger than $5 \cdot 10^{-3}$.

By looking at Figure 7, we observe that for $\mathrm{SNR}_{m}=30 \mathrm{~dB}$ it is sufficient to have $\eta^{2}<10$ so as to provide the required MSE. Interestingly, we note that a perfectly equally spaced (and very expensive) deployment, i.e., $\eta^{2}=0$, does not provide an MSE lower than $2 \cdot 10^{-3}$.

\section{CONCLUSIONS}

We considered randomly deployed sensor networks sampling a bandlimited field, and we analyzed their performance in terms of the MSE obtained on the reconstructed field. In particular, by using asymptotic analysis, we studied the case of uniformly distributed sensors and then we focused on quasi-equally spaced sensor layouts. For the latter, we were able to derive approximate closed form expressions of the MSE, which was shown to be very tight. Finally, we gave an example of how useful our results can be for the design of sensor networks.

\section{REFERENCES}

[1] H. G. Feichtinger, K. Gröchenig, T. Strohmer, "Efficient numerical methods in non-uniform sampling theory," Numerische Mathematik, Vol. 69, 1995, pp. 423-440.

[2] T. Strohmer, "Efficient Methods for Digital Signal and Image Reconstruction from Nonuniform Samples.", PhD thesis, University of Vienna, 1993. 
[3] T. Strohmer, T. Binder and M. Süssner, "How to recover smooth object boundaries in noisy medical images", IEEE ICIP'96, pp.331-334, Lausanne, 1996.

[4] M. Rauth and T. Strohmer, "A frequency domain approach to the recovery of geophysical potentials," Proc. Conf. SampTA'97, pp.109-114, Aveiro/Portugal, 1997.

[5] R. Daley, "Atmospheric Data Analysis," Cambridge Atmospheric and Space Science Series, 1991.

[6] I. F. Akyildiz, W. Su, Y. Sankarasubramaniam, and E. Cayirci, "Wireless sensor networks: A survey," Computer Networks, vol.38, no. 4, pp. 393-422, 2002.

[7] D. Ganesan, S. Ratnasamy, H. Wang, and D. Estrin, "Coping with irregular spatio-temporal sampling in sensor networks", ACM SIGCOMM vol. 34, no. 1, pp.125-130, January 2004.

[8] P.Zhao, c. Zhao, ans P.G. Casazza, "Perturbation of Regular Sampling in Shift-Invariant Spaces for Frames" IEEE Transactions on Information Theory, vol. 52, no. 10, pp.4643-4648, October 2006.

[9] D.S. Early and D.G. Long, "Image reconstruction and enhanced resolution imaging from irregular samples," IEEE Transactions on Geoscience and Remote Sensing, vol. 39, no. 2, pp.291-302, February 2001.

[10] A. Nordio, C-F. Chiasserini, and E. Viterbo, "Bandlimited Field Reconstruction for Wireless Sensor Networks" Tech. Rep., Politecnico di Torino, January 2006.

[11] A. Nordio, C-F. Chiasserini, and E. Viterbo, "Performance of Field Reconstruction Techniques with Noise and Uncertain Sensor Locations" IEEE Transactions on Signal Processing, submitted, October 2006.

[12] J. Hightower, G. Borriello, "Location systems for ubiquitous computing," IEEE Computer, vol. 34, no. 8, pp.57-66, Aug. 2001.

[13] L. Hu, D. Evans, "Localization for mobile sensor networks," ACM MobiCom 2004, Philadelphia, PA, Sept.-Oct. 2004.

[14] D. Moore, J. Leonard, D. Rus, S. Teller, "Robust distributed network localization with noisy range measurements," 2nd ACM Conference on Embedded Networked Sensor Systems (SenSys '04), Baltimore, MD, pp. 50-61, Nov. 2004.

[15] G. Strang, "Wavelet Transforms Versus Fourier Transforms," Bull. Amer. Math. Soc. 28, no. 2, pp. 288-305, 1993.

[16] S. M. Kay, Fundamentals of Statistical Signal Processing: Estimation Theory, Prentice Hall, 1993.

[17] A. Tulino and S. Verdú, "Random Matrix Theory and Wireless Communications," Foundations and Trends in Communications and Information Theory, vol. 1, no. 1, 2004.

[18] E. W. Weisstein, "Euler-Mascheroni Constant," from MathWorld - A Wolfram Web Resource. http://mathworld.wolfram.com/EulerMascheroniConstant.html

\section{APPENDIX}

\section{A. PROOFS OF (11)-(13)}

Here we provide the proof of claims (11)-(13). Concerning (11), from the definition of $\phi(\cdot)$, we have

$$
\phi\left(\mathbf{R}_{\mathbf{x}}^{p}\right)=\lim _{M, r \rightarrow+\infty} \frac{1}{2 M+1} \mathbb{E}\left[\operatorname{Tr}\left\{\mathbf{R}_{\mathbf{x}}^{p}\right\}\right]
$$

Expanding the matrix power, we obtain:

$$
\begin{aligned}
& \mathbb{E}\left[\operatorname{Tr}\left\{\mathbf{R}_{\mathbf{x}}^{p}\right\}\right]=\mathbb{E}\left[\sum_{\ell_{1}=-M}^{M}\left(\mathbf{R}_{\mathbf{x}}^{p}\right) \ell_{1} \ell_{1}\right] \\
& =\mathbb{E}\left[\sum_{1}\left(\mathbf{R}_{\mathbf{x}}\right) \ell_{1} \ell_{2}\left(\mathbf{R}_{\mathbf{x}}\right) \ell_{2} \ell_{3} \cdots\left(\mathbf{R}_{\mathbf{x}}\right) \ell_{p-1} \ell_{p}\left(\mathbf{R}_{\mathbf{x}}\right) \ell_{p} \ell_{1}\right]
\end{aligned}
$$

where $\mathrm{l}=\left[\ell_{1}, \ldots, \ell_{p}\right]$ is a vector of integers, and $\ell_{i} \in\{-M, \ldots,+M\}$, $i=1, \ldots, p$. Since $\mathbf{R}_{\mathbf{x}}=\mathbf{G}_{\mathbf{x}} \mathbf{G}_{\mathbf{x}}^{\dagger}$, the $k h$-th entry of $\mathbf{R}_{\mathbf{x}}$ is given by:

$$
\left(\mathbf{R}_{\mathbf{x}}\right)_{k h}=\frac{1}{2 M+1} \sum_{q=1}^{r} \mathrm{e}^{-\mathrm{j} 2 \pi(k-h) x_{q}}
$$

Thus,

$$
\begin{aligned}
& \mathbb{E}\left[\operatorname{Tr}\left\{\mathbf{R}_{\mathbf{x}\}}^{p}\right\}\right] \\
& =\frac{1}{(2 M+1)^{p}} \sum_{1} \sum_{q} \mathbb{E}\left[\mathrm{e}^{-j 2 \pi\left(\ell_{1}-\ell_{2}\right) x_{q_{1}}-\cdots-j 2 \pi\left(\ell_{p}-\ell_{1}\right) x_{q_{P}}}\right](22)
\end{aligned}
$$

and similarly,

$$
\begin{aligned}
& \mathbb{E}\left[\operatorname{Tr}\left\{\mathbf{R}_{\hat{\mathbf{x}}}^{p}\right\}\right] \frac{1}{(2 M+1)^{p}} \sum_{\mathbf{I}} \sum_{\mathbf{q}} \mathbb{E}\left[\mathrm{e}^{-\mathrm{j} 2 \pi\left(\ell_{1}-\ell_{2}\right) \hat{x}_{q_{1}}-\cdots-\mathrm{j} 2 \pi\left(\ell_{p}-\ell_{1}\right) \hat{x}_{q_{p}}}\right] \\
&= \frac{1}{(2 M+1)^{p}} \sum_{\mathbf{I}} \sum_{\mathbf{q}} \underset{\mathbf{x}}{\mathbb{E}}\left[\mathrm{e}^{-\mathrm{j} 2 \pi\left(\ell_{1}-\ell_{2}\right) x_{q_{1}}-\cdots-\mathrm{j} 2 \pi\left(\ell_{p}-\ell_{1}\right) x_{q_{p}}}\right] \\
& \frac{\mathbb{E}}{\delta}\left[\mathrm{e}^{-\mathrm{j} 2 \pi\left(\ell_{1}-\ell_{2}\right) \delta_{q_{1}}-\cdots-\mathrm{j} 2 \pi\left(\ell_{p}-\ell_{1}\right) \delta_{q_{p}}}\right]
\end{aligned}
$$

where $\mathrm{q}=\left[q_{1}, \ldots, q_{p}\right]$ is a vector of integers with $q_{j} \in\{1, \ldots, r\}$, $j=1, \ldots, p$. In practice (23) is obtained by summing products of averages of the type $\underset{x}{\mathbb{E}}\left[\mathrm{e}^{-\mathrm{j} 2 \pi k x}\right] \underset{\delta}{\mathbb{E}}\left[\mathrm{e}^{-\mathrm{j} 2 \pi k \delta}\right]$, for any integer $k$. Notice that

$$
\underset{x}{\mathbb{E}_{s}}\left[\mathrm{e}^{-\mathrm{j} 2 \pi k x}\right] \underset{\delta}{\underset{\delta}{\mathbb{F}_{\delta}}}\left[\mathrm{e}^{-\mathrm{j} 2 \pi k \delta}\right]=C_{x}(-\mathrm{j} 2 \pi k) C_{\delta}(-\mathrm{j} 2 \pi k)
$$

where $C_{x}(s)$ and $C_{\delta}(s)$ are the characteristic functions of the random variables $x$ and $\delta$, respectively. Since $x$ is uniformly distributed in $[-1 / 2,+1 / 2)$, then

$$
\begin{aligned}
C_{x}(s) & =\int_{-\infty}^{+\infty} \mathrm{e}^{s x} f_{\lambda}(x, \beta) \mathrm{d} x \\
& =\int_{-1 / 2}^{+1 / 2} \mathrm{e}^{s x} \mathrm{~d} x=\frac{\mathrm{e}^{s / 2}-\mathrm{e}^{-s / 2}}{s}
\end{aligned}
$$

Therefore $C_{x}(-\mathrm{j} 2 \pi k)=1$ for $k=0$, and $C_{x}(-\mathrm{j} 2 \pi k)=0$ for $k \neq 0$. It follows that

$$
C_{x}(-\mathrm{j} 2 \pi k) C_{\delta}(-\mathrm{j} 2 \pi k)=C_{x}(-\mathrm{j} 2 \pi k) C_{\delta}(0)=C_{x}(-\mathrm{j} 2 \pi k)
$$

since $C_{\delta}(0)=1$ by definition of the characteristic function. It turns out that

$$
\underset{x}{\mathbb{E}}\left[\mathrm{e}^{-\mathrm{j} 2 \pi k x}\right] \underset{\delta}{\mathbb{E}}\left[\mathrm{e}^{-\mathrm{j} 2 \pi k \delta}\right]=\mathbb{E}\left[\mathrm{e}^{-\mathrm{j} 2 \pi k x}\right]
$$

and by consequence $\mathbb{E}\left[\operatorname{Tr}\left\{\mathbf{R}_{\hat{\mathbf{x}}}^{p}\right\}\right]=\mathbb{E}\left[\operatorname{Tr}\left\{\mathbf{R}_{\mathbf{x}}^{p}\right\}\right]$, so that $\phi\left(\mathbf{R}_{\hat{\mathbf{x}}}^{p}\right)=$ $\phi\left(\mathbf{R}_{\mathbf{x}}^{p}\right)$.

The proof of (12) and (13) can be given in a similar way. 


\section{B. PROOF OF (14) AND (15)}

From the definition in (2), we have:

$$
\begin{aligned}
\frac{\mathbb{E}}{\delta}\left[\left(\mathbf{G}_{\hat{\mathbf{x}}}\right)_{k q}\right] & =\frac{1}{\sqrt{2 M+1}} \frac{\mathbb{E}}{\delta}\left[\mathrm{e}^{-\mathrm{j} 2 \pi k \hat{x}_{q}}\right] \\
& =\frac{1}{\sqrt{2 M+1}} \mathrm{e}^{-\mathrm{j} 2 \pi k x_{q}} \underset{\delta}{\mathbb{E}\left[\mathrm{e}^{-\mathrm{j} 2 \pi k \delta_{q}}\right]} \\
& =\frac{1}{\sqrt{2 M+1}} \mathrm{e}^{-\mathrm{j} 2 \pi k x_{q}} C_{\delta}(-\mathrm{j} 2 \pi k) \\
& =(\mathbf{C})_{k k}\left(\mathbf{G}_{\mathbf{x}}\right)_{k q}
\end{aligned}
$$

where $C_{\delta}(s)=\int_{-\infty}^{+\infty} \exp (s x) f_{\delta}(x) \mathrm{d} x$ is the characteristic function of $\delta$ and $\mathbf{C}$ is a diagonal matrix with entries given by $(\mathbf{C})_{k k}=$ $C_{\delta}(-\mathrm{j} 2 \pi k)$. We conclude that:

$$
\underset{\delta}{\mathbb{E}}\left[\mathrm{G}_{\hat{\mathbf{x}}}\right]=\mathrm{CG}_{\mathbf{x}}
$$

Similarly,

$$
\begin{aligned}
\underset{\delta}{\mathbb{E}}\left[\left(\mathbf{G}_{\hat{\mathbf{x}}}^{\dagger} \mathbf{G}_{\hat{\mathbf{x}}}\right)_{q q^{\prime}}\right] \\
\quad=\frac{1}{2 M+1} \sum_{k=-M}^{+M} \mathbb{\delta}\left[\mathrm{e}^{\mathrm{j} 2 \pi k \hat{x}_{q}} \mathrm{e}^{\left.-\mathrm{j} 2 \pi k \hat{x}_{q^{\prime}}\right]}\right. \\
\quad=\frac{1}{2 M+1} \sum_{k=-M}^{+M} \mathrm{e}^{\mathrm{j} 2 \pi k\left(x_{q}-x_{q^{\prime}}\right)} \underset{\delta}{\mathbb{\delta}\left[\mathrm{e}^{\mathrm{j} 2 \pi k\left(\delta_{q}-\delta_{q^{\prime}}\right)}\right]}
\end{aligned}
$$

If $q \neq q^{\prime}$,

$$
\begin{aligned}
& \underset{\delta}{\underset{\delta}{\mathbb{E}}}\left[\left(\mathbf{G}_{\mathbf{x}}^{\dagger} \mathbf{G}_{\mathbf{x}}\right)_{q q^{\prime}}\right] \\
& =\frac{1}{2 M+1} \sum_{k=-M}^{+M} \mathrm{e}^{\mathrm{j} 2 \pi k\left(x_{q}-x_{q^{\prime}}\right)} \underset{\delta}{\mathbb{E}}\left[\mathrm{e}^{\mathrm{j} 2 \pi k \delta_{q}}\right] \underset{\delta}{\mathbb{E}}\left[\mathrm{e}^{-\mathrm{j} 2 \pi k \delta_{q^{\prime}}}\right] \\
& =\frac{1}{2 M+1} \sum_{k=-M}^{+M} \mathrm{e}^{\mathrm{j} 2 \pi k\left(x_{q}-x_{q^{\prime}}\right)} C_{\delta}(\mathrm{j} 2 \pi k) C_{\delta}(-\mathrm{j} 2 \pi k) \\
& =\left(\mathbf{G}_{\mathbf{x}}^{\dagger} \mathbf{C}^{\dagger} \mathbf{C G}_{\mathbf{x}}\right)_{q q^{\prime}}
\end{aligned}
$$

If $q=q^{\prime}$,

$$
\begin{aligned}
\underset{\delta}{\mathbb{E}}[ & \left.\left(\mathbf{G}_{\hat{\mathbf{x}}}^{\dagger} \mathbf{G}_{\hat{\mathbf{x}}}\right)_{q q}\right] \\
& =\frac{1}{2 M+1} \sum_{k=-M}^{+M} \mathrm{e}^{\mathrm{j} 2 \pi k\left(x_{q}-x_{q}\right)} \underset{\delta}{\mathbb{E}}\left[\mathrm{e}^{\mathrm{j} 2 \pi k\left(\delta_{q}-\delta_{q}\right)}\right] \\
& =\frac{1}{2 M+1} \sum_{k=-M}^{+M} 1 \\
& =1
\end{aligned}
$$

Since $\left(\mathbf{G}_{\mathbf{x}}^{\dagger} \mathbf{C}^{\dagger} \mathbf{C} \mathbf{G}_{\mathbf{x}}\right)_{q q}=\operatorname{Tr}\left\{\mathbf{C}^{\dagger} \mathbf{C}\right\}$, we obtain:

$$
\underset{\delta}{\mathbb{E}}\left[\mathbf{G}_{\hat{\mathbf{x}}}^{\dagger} \mathbf{G}_{\hat{\mathbf{x}}}\right]=\mathbf{G}_{\mathbf{x}}^{\dagger} \mathbf{C}^{\dagger} \mathbf{C G}_{\mathbf{x}}+\left(1-\frac{\operatorname{Tr}\left\{\mathbf{C}^{\dagger} \mathbf{C}\right\}}{2 M+1}\right) \mathbf{I}
$$

